\title{
Development of a non-invasive fibrosis test for chronic hepatitis B patients and comparison with other unpatented scores
}

\author{
Chao-Wei Hsu' ${ }^{1+}$, Kung-Hao Liang ${ }^{1 \dagger}$, Shiu-Feng Huang ${ }^{2}$, Kuo-Chien Tsao ${ }^{3,4}$ and Chau-Ting Yeh ${ }^{1 *}$
}

\begin{abstract}
Background: Despite the availability of patented non-invasive methods, evaluation of the degrees of liver fibrosis remains difficult when conducting a retrospective study. Such inadequacy is largely caused by requirement of biochemical parameters rarely performed in routine clinical tests. We developed a novel fibrosis HB-F score using commonly performed tests for HBV infected patients.

Methods: 424 patients with chronic HBV infection were included. Using clinical and virological data, HB-F score was developed from a training cohort $(n=213)$ and validated in a separate cohort $(n=211)$. The performance was compared with five other unpatented scores using ROC curves.

Results: Univariate and multivariate analysis revealed that age, AST/ALT ratio, platelet count and prothrombin time prolongation were significantly associated with the ISHAK fibrosis score, and were used to calculate the HB-F score. When HB-F was used to assess prominent fibrosis and cirrhosis, the AUC was 0.81 and 0.80 respectively in the training cohort, and 0.80 and 0.76 respectively in the validation cohort. HB-F had the highest AUC compared with other scores. Furthermore, in assessing paired liver biopsies with increase or decrease of ISHAK scores, HB-F showed significant change in the same direction.
\end{abstract}

Conclusions: A new non-invasive score was developed, which could be used to assess severity of liver fibrosis in retrospective longitudinal studies in HBV patients.

Keywords: Hepatitis B, Fibrosis, Score, Receiver operation characteristic curve

\section{Background}

It has been estimated that 350 millions patients are infected by hepatitis B virus (HBV) worldwide [1]. Chronic HBV infection may lead to severe sequelae such as liver fibrosis and cirrhosis [1]. Liver fibrosis is a progressive damage which not only impairs liver functions but also increases the risk of hepatocellular carcinoma [2]. The progression of fibrosis has multiple stages which are commonly defined by ISHAK or METAVIR scoring systems [3-5]. An advanced fibrosis score represents a serious clinical condition which requires careful medical managements. To prevent disease progression, we need to detect liver fibrosis at an earlier stage and to

\footnotetext{
* Correspondence: chautingy@gmail.com

${ }^{\dagger}$ Equal contributors

'Liver Research Center, Chang Gung Memorial Hospital, 199, Tung Hwa North Road, Taipei, Taiwan

Full list of author information is available at the end of the article
}

provide adequate antiviral agent treatment [6]. In the past decades, liver biopsy has been a gold standard for the assessment of fibrosis stages. However, there are several limitations when applying this method, such as sampling bias, low platelet counts, prolonged prothrombin time, massive ascites, patient's intention and compliance as well as scoring variations from different pathologists [7]. Thus, other methods to accurately assess liver fibrosis are continuously sought, especially for non-invasive tests.

Ultrasound is one possible alternative for non-invasive assessment. FibroScan and Acoustic radiation force impulse (ARFI) elastography have been used for evaluation of fibrosis in chronic hepatitis B or C patients. However, it was found that the accuracy of FibroScan and ARFI was greatly interfered by higher degrees of necroinflammation

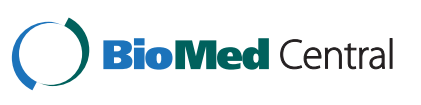

(c) 2013 Hsu et al.; licensee BioMed Central Ltd. This is an Open Access article distributed under the terms of the Creative Commons Attribution License (http://creativecommons.org/licenses/by/2.0), which permits unrestricted use, distribution, and reproduction in any medium, provided the original work is properly cited. 
and more advanced stages of fibrosis or cirrhosis especially in chronic hepatitis B patients [8-11].

Recently, a number of fibrosis scores have been proposed as surrogates to liver biopsy. These scores are often composed of a combination of biochemistry measurements and clinical parameters. For example, the AAR score is based on the ratio of aspartate transaminase (AST) and alanine transaminase (ALT) values [12]. The APRI score is the AST value divided by platelet counts [13]. The early AAR and APRI scores motivate several subsequent scores where the ratios of AST/ALT or AST/ platelet counts are employed as part of their equations. The Fibrosis index (FI) score is composed of platelet counts and serum albumin [14]. The Fibroindex consists of AST, platelets, and gamma globulin measurements [15]. The FIB-4 index includes age, AST, ALT and platelet counts [16,17]. A patented test, named FibroTest, is composed of alpha2-macroglobulin, haptoglobin, gamma glutamyl transpeptidase (GGT), age, bilirubin, apoA1, and sex. Most of these markers were initially derived from patients with chronic hepatitis $\mathrm{C}$ but were subsequently tested in chronic hepatitis B patients. Meta-analysis for the performance of biomarkers in $\mathrm{HBV}$ or hepatitis $\mathrm{C}$ virus (HCV) infection showed that assessment of the treatment efficacy on fibrosis progression was equally effective when estimated by either FibroTest or biopsy. Despite the great performance of FibroTest, there were still no sufficient data to show that biomarker or biopsy alone could make accurate fibrosis staging in patients with chronic HBV infection [18]. Furthermore, the requirement of measuring several uncommon tests to calculate the score greatly limited its use in retrospective study [19-21]. We were thus motivated to formulate a new hepatitis B-fibrosis score (HB-F) using commonly performed tests and clinical data in a large cohort of chronic HBV infected patients.

\section{Methods}

\section{Patients and samples}

Under approval of Institutional Review Board, Chang Gung Medical Council, this study was conducted at Liver Research Center, Chang Gung Memorial Hospital, Taiwan. A total of 424 adult patients with compensated chronic hepatitis B were recruited (Table 1). All of them have signed informed consent forms. They received liver biopsy between January 2007 and July 2009. No antiviral agents were given to these patients before biopsy was taken and patients with other viral co-infections, such as HIV, hepatitis C or hepatitis D co-infections were preexcluded. Hepatitis B e Antigen ( $\mathrm{HBeAg}$ ) and antibodies to $\mathrm{HBeAg}$ (anti-HBe) were measured before liver biopsy. Biochemistry were assessed at the same time of liver biopsy, including AST, ALT, bilirubin, gamma glutamyl transpeptidase (GGT), albumin, alpha-1 globulin, alpha-
Table 1 Basic clinicopathological data for hepatitis B patients included

\begin{tabular}{|c|c|c|}
\hline Characteristics & $\begin{array}{l}\text { Training cohort } \\
(\mathbf{n}=213)\end{array}$ & $\begin{array}{l}\text { Verification } \\
\text { cohort }(n=211)\end{array}$ \\
\hline $\begin{array}{l}\text { Age at biopsy in years, } \\
\text { mean } \pm \text { SD }\end{array}$ & $44.4 \pm 11.8$ & $45.6 \pm 11.4$ \\
\hline Male, n (\%) & $179(84)$ & $175(83)$ \\
\hline \multicolumn{3}{|l|}{ Biopsy } \\
\hline \multicolumn{3}{|l|}{$\begin{array}{l}\text { Necroinflammation score, } \\
\text { mean } \pm \text { SD }\end{array}$} \\
\hline Periportal inflammation & $1.0 \pm 0.8$ & $0.9 \pm 0.8$ \\
\hline Confluence necrosis & $0.3 \pm 1.0$ & $0.2 \pm 0.8$ \\
\hline Focal inflammation & $1.9 \pm 0.7$ & $2.0 \pm 0.7$ \\
\hline Portal inflammation & $2.2 \pm 1.0$ & $2.2 \pm 0.9$ \\
\hline \multicolumn{3}{|l|}{ ISHAK fibrosis score, n (\%) } \\
\hline 0 & $1(1)$ & $1(1)$ \\
\hline 1 & $28(13)$ & $28(13)$ \\
\hline 2 & $32(15)$ & $32(15)$ \\
\hline 3 & $70(33)$ & $71(34)$ \\
\hline 4 & $21(10)$ & $21(10)$ \\
\hline 5 & $46(22)$ & $45(21)$ \\
\hline 6 & $15(7)$ & $13(6)$ \\
\hline HBeAg-positive, n (\%) & $109(51)$ & $91(43)$ \\
\hline \multicolumn{3}{|l|}{ Biochemistry, mean \pm SD } \\
\hline AST (IU/mL) & $91.3 \pm 83.6$ & $94.9 \pm 100.1$ \\
\hline ALT (IU/mL) & $170.1 \pm 173.8$ & $166.2 \pm 177.9$ \\
\hline AST/ALT & $0.6 \pm 0.3$ & $0.6 \pm 0.3$ \\
\hline AFP $(n g / m L)$ & $10.5 \pm 22.2$ & $20.4 \pm 82.0$ \\
\hline Bilirubin (mg/dL) & $1.0 \pm 0.7$ & $1.0 \pm 0.4$ \\
\hline GGT (IU/L) & $69.0 \pm 77.0$ & $60.1 \pm 57.3$ \\
\hline Albumin (g/dL) & $4.6 \pm 0.3$ & $4.5 \pm 0.4$ \\
\hline Platelet $\left(\times 10^{3} / \mathrm{mm}^{3}\right)$ & $188.2 \pm 54.2$ & $189.2 \pm 49.4$ \\
\hline $\begin{array}{l}\text { Prothrombin time } \\
\text { prolongation (sec) }\end{array}$ & $1.0 \pm 0.6$ & $1.0 \pm 0.8$ \\
\hline White blood cell $\left(\times 10^{3} / \mathrm{mm}^{3}\right)$ & $5.7 \pm 1.7$ & $5.5 \pm 1.6$ \\
\hline Hemoglobin (g/dL) & $15.1 \pm 1.4$ & $15.2 \pm 1.3$ \\
\hline Alpha-1 globulin (g/dL) & $0.2 \pm 0.1$ & $0.2 \pm 0.0$ \\
\hline Alpha-2 globulin (g/dL) & $0.8 \pm 0.2$ & $0.8 \pm 0.1$ \\
\hline Beta globulin (g/dL) & $0.8 \pm 0.1$ & $0.7 \pm 0.1$ \\
\hline Gamma globulin (g/dL) & $1.5 \pm 0.4$ & $1.4 \pm 0.4$ \\
\hline Albumin/Globulin & $1.5 \pm 0.3$ & $1.5 \pm 0.3$ \\
\hline
\end{tabular}

2 globulin, beta globulin, gamma globulin and albumin/ globulin ratio. Hematology tests were also performed at same time point, including hemoglobin, platelet count, white blood cell, prothrombin time, and alphafetoprotein (AFP).

Histologic evaluation of biopsy samples were carried out at the Pathology Department, according to ISHAK's 
staging system, which had seven severity levels: FS0 (no fibrosis), FS1 (fibrous expansion of some portal areas), FS2 (fibrous expansion of most portal areas), FS3 (fibrous expansion of most portal areas with occasional portal to portal bridging), FS4 (fibrous expansion of most portal areas with marked bridging), FS5 (marked bridging with occasional nodules, incomplete cirrhosis), and FS6 (cirrhosis).

After biopsy, subjects of each ISHAK stage were randomly assigned into two cohorts: the training cohort $(\mathrm{n}=213)$ and the validation cohort $(\mathrm{n}=211)$, for formuladeriving and validation purposes respectively. This way, subjects were evenly split across all ISHAK stages. Clinical parameters and biochemistry measurements were individually tested for their association to the ISHAK fibrosis stages. The associated factors were then combined to produce the HB-F score. The score was then validated using the validation cohort, and its performance was compared with five other reported scores (AAR, FIB-4, FI, APRI, Fibroindex), which were all originally derived from hepatitis $C$ infected patients.

\section{Statistical analysis}

Clinicopathological data distribution was compared between the training and validation cohorts by either Chi-square tests or two-sample t-tests with unequal variance. Linear regression was used for the univariate and multivariate analysis to assess the association between the clinical/ laboratory parameters and ISHAK fibrosis scores. Significance levels of correlation were assessed by Wald test statistics. All the P-values were two-tailed. The Receiver Operating Characteristic (ROC) Curve was used to examine the trade-off of sensitivity and specificity. The performance of classification was assessed by the Area Under Curve (AUC). The HB-F and FIB-4 scores of the two biopsy examinations were assessed by paired $t$-tests.

\section{Results}

The distributions of subjects' clinicopathological values were presented in Table 1. The two cohorts had similar number of subjects across all ISHAK stages without significant disparity $(\mathrm{P}=1.000)$. Additionally, comparison of the age, gender, inflammation scores and the biochemistry and hemogram values revealed no significant difference.

Univariate and multivariate associations of various factors to ISHAK fibrosis stages were performed in the training cohort (Table 2). Significant associations were found in eight parameters, including age at biopsy, HBeAg, AST/ALT ratio, AFP, GGT, Albumin, platelet and prothrombin time prolongation $(\mathrm{P}<0.05)$. Among them, four parameters (age at biopsy, AST/ALT ratio, platelet and prothrombin time prolongation) showed stronger association $(\mathrm{P}<0.001)$. A multivariate analysis of the four parameters showed that all of them remained significant $(P<0.05)$, indicating their independent association to the ISHAK fibrosis stages.

Table 2 Univariate and multivariate analysis for factors associated with liver fibrosis

\begin{tabular}{|c|c|c|c|c|}
\hline Characteristics & Beta $(95 \% \mathrm{Cl})$ & $\mathbf{P}$ & Adjusted beta $(95 \% \mathrm{Cl})$ & $P$ \\
\hline Age at biopsy & $0.035(0.018,0.052)$ & $<0.001$ & $0.018(0.001,0.036)$ & 0.044 \\
\hline Male & $0.046(-0.597,0.506)$ & 0.870 & & \\
\hline HBeAg-positive & $-0.496(-0.943,-0.048)$ & 0.030 & & \\
\hline AST (IU/mL) & $0.001(-0.001,0.004)$ & 0.363 & & \\
\hline ALT $(I \cup / m L)$ & $0.000(-0.001,0.001)$ & 0.802 & & \\
\hline AST/ALT & $1.881(1.199,2.563)$ & $<0.001$ & $1.085(0.368,1.803)$ & 0.003 \\
\hline $\operatorname{AFP}(\mathrm{ng} / \mathrm{mL})$ & $0.012(0.001,0.023)$ & 0.026 & & \\
\hline Bilirubin (mg/dL) & $0.142(-0.168,0.452)$ & 0.367 & & \\
\hline GGT (IU/L) & $0.006(0.002,0.009)$ & 0.003 & & \\
\hline Albumin (g/dL) & $-1.440(-2.839,-0.042)$ & 0.044 & & \\
\hline Platelet $\left(\times 10^{3} / \mathrm{mm}^{3}\right)$ & $-0.012(-0.015,-0.009)$ & $<0.001$ & $-0.009(-0.013,-0.005)$ & $<0.001$ \\
\hline Prothrombin time prolongation (sec) & $0.781(0.404,1.157)$ & $<0.001$ & $0.449(0.098,0.800)$ & 0.012 \\
\hline White blood cell $\left(\times 10^{3} / \mathrm{mm}^{3}\right)$ & $0.049(-0.093,0.192)$ & 0.495 & & \\
\hline Hemoglobin (g/dL) & $0.053(-0.125,0.231)$ & 0.558 & & \\
\hline Alpha-1 globulin (g/dL) & $-0.898(-2.683,0.886)$ & 0.322 & & \\
\hline Alpha-2 globulin (g/dL) & $-0.505(-1.746,0.736)$ & 0.423 & & \\
\hline Beta globulin (g/dL) & $-0.808(-2.160,0.545)$ & 0.240 & & \\
\hline Gamma globulin (g/dL) & $0.214(-0.327,0.754)$ & 0.436 & & \\
\hline Albumin/Globulin & $-0.422(-1.180,0.335)$ & 0.273 & & \\
\hline
\end{tabular}


The four parameters were then linearly combined to generate the HB-F score using the multiple regression coefficients (Adjusted Beta) in Table 2, omitting the constant term. The formula of HB-F score was written as:

$\mathrm{HB}-\mathrm{F}=0.018 \times[$ Age $]+1.085 \times[$ AST $/$ ALT $]-0.009 \times$ [Platelet $\left.\left(\times 10^{3} / \mathrm{mm}^{3}\right)\right]+0.449 \times[$ Prothrombin time prolongation $(\mathrm{sec})]$

In the training cohort, subjects with a more severe fibrosis stage also had a higher range of HB-F score (Figure 1). The score was then used to classify patients with (FS $>=5$ ) or without cirrhosis $(\mathrm{FS}<5)$ and patients with $(F S>=4)$ or without severe fibrosis $(F S<4)$. The AUCs were 0.810 and 0.799 for the distinction of severe fibrosis and cirrhosis, respectively, in the training cohort. Consistently, the corresponding AUCs were 0.797 and 0.757 respectively in the verification cohort. Both AUCs were larger than 0.75 suggesting adequate capability of HB-F in identifying severe fibrosis subjects (Figure 1).
When compared with five other reported methods, HB-F had the highest AUC (Table 3, Figure 2). The next three best scoring methods were FIB-4, Fibroindex and APRI, respectively, but all had no statistically significant difference when compared with HB-F score. AAR, on the other hand, had a significantly inferior performance in classifying both the cirrhosis and prominent fibrosis. FI had an inferior performance in identifying prominent fibrosis. The ROCs of all the methods were presented in Figure 2.

A total of 33 subjects had received a second biopsy and biochemistry examinations. They offered additional disease progression and remission data for evaluating the change of HB-F and FIB-4 score under an increase or decrease of ISHAK levels (Figure 3). FIB-4 was chosen because it had the highest AUC among the benchmark scores. The HB-F scores showed significant changes in the same direction of an increment $(\mathrm{P}=0.011)$ and decrement $(\mathrm{P}=0.015)$ of ISHAK levels. In contrast, FIB-4 did not show such a correlated change.
A

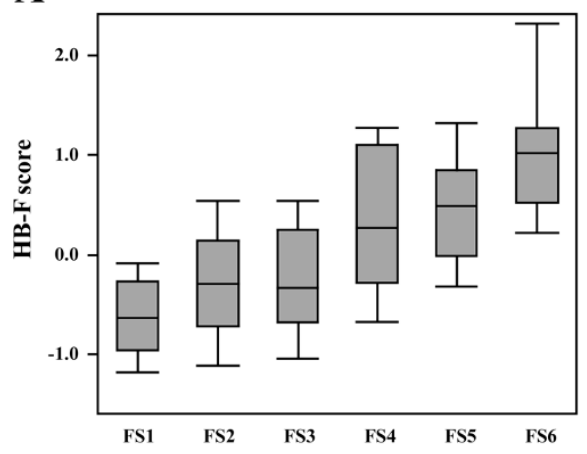

D

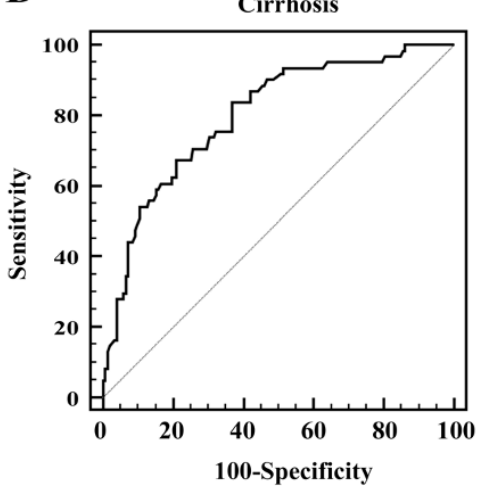

B

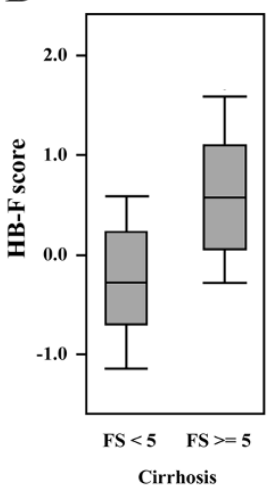

C

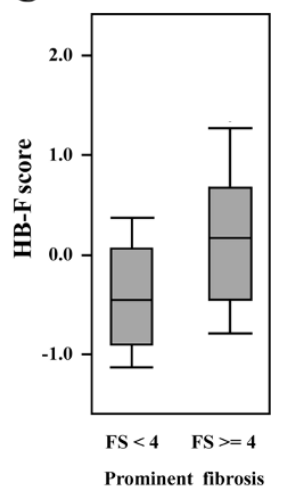

$\mathbf{E}$

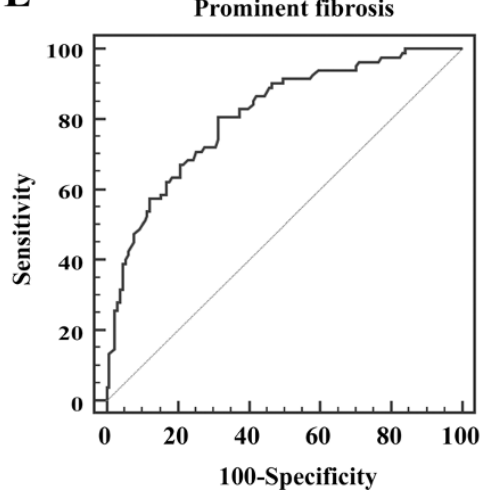

Figure 1 Performance of the HB-F fibrosis score in the training cohort of patients. (A) The ranges of HB-F scores in patients with different ISHAK fibrosis score (FS1-FS6). Gray boxes represented the 25\%-75\% quartiles; The middle horizontal lines represented the median scores. (B) The ranges of HB-F scores in subjects stratified by FS $<5$ (non-cirrhotic patients) and FS $>=5$ (cirrhosis) $(\mathrm{P}<0.001)$. (C) The ranges of HB-F scores in subjects grouped by FS $<4$ and FS $>=4$ (prominent fibrosis) $(P<0.001$ ). (D) The ROC curve of the HB-F score for the diagnosis of cirrhosis patients ( $A \cup C=0.799)$. $(E)$ The ROC curve for the diagnosis of prominent fibrosis patients $(A \cup C=0.810)$. 
Table 3 Performance of six fibrosis score for evaluation of liver fibrosis and cirrhosis in patients with chronic hepatitis B

\begin{tabular}{|c|c|c|c|}
\hline Non-invasive index & AUC $(95 \% \mathrm{Cl})$ & Difference between areas $(95 \% \mathrm{Cl})^{*}$ & $\mathbf{P}^{*}$ \\
\hline \multicolumn{4}{|l|}{ Prominent fibrosis } \\
\hline HB-F (training) & $0.810(0.751,0.861)$ & & \\
\hline HB-F (verification) & $0.797(0.737,0.849)$ & & \\
\hline FIB-4 & $0.772(0.709,0.826)$ & $0.0255(-0.0236,0.0746)$ & 0.3087 \\
\hline Fibroindex & $0.767(0.704,0.822)$ & $0.0301(-0.0298,0.0899)$ & 0.3251 \\
\hline ARPI & $0.753(0.689,0.810)$ & $0.0439(-0.0302,0.1180)$ & 0.2454 \\
\hline AAR & $0.630(0.561,0.696)$ & $0.1670(0.0947,0.2390)$ & $<0.0001$ \\
\hline $\mathrm{Fl}$ & $0.724(0.659,0.784)$ & $0.0728(0.0093,0.1360)$ & 0.0246 \\
\hline \multicolumn{4}{|l|}{ Cirrhosis } \\
\hline HB-F (training) & $0.799(0.739,0.851)$ & & \\
\hline HB-F (verification) & $0.757(0.694,0813)$ & & \\
\hline F|B-4 & $0.727(0.662,0.786)$ & $0.0297(-0.0223,0.0818)$ & 0.2624 \\
\hline Fibroindex & $0.722(0.656,0.781)$ & $0.0351(-0.0320,0.1020)$ & 0.3054 \\
\hline ARPI & $0.698(0.631,0.759)$ & $0.0589(-0.0244,0.1400)$ & 0.1556 \\
\hline AAR & $0.636(0.568,0.701)$ & $0.1210(0.0470,0.1950)$ & 0.0013 \\
\hline $\mathrm{FI}$ & $0.717(0.651,0.777)$ & $0.0403(-0.0283,0.1090)$ & 0.2497 \\
\hline
\end{tabular}

*Comparison between the $A U C$ of $H B-F$ (verification cohort) and those of the other scoring methods.

\section{Discussion}

In this study, a novel fibrosis score named HB-F was formulated, by combination of four factors which was highly significant in the association with ISHAK fibrosis stages $(\mathrm{P}<0.001)$. The HB-F score was developed from a large cohort of $\mathrm{HBV}$-infected patients, as HBV infection is a major etiology of fibrosis in Taiwan. Although the study also included other $\mathrm{HBV}$-related factors such as the presence or absence of $\mathrm{HBeAg}$, this factor only manifested marginal association to the ISHAK stages $(\mathrm{P}=0.03)$. Hence, the HBV-related factors were not included in HB-F.

The four factors in HB-F score included age, the AST/ ALT ratio from biochemistry test, platelet count and prothrombin time prolongation from hematological test. The prothrombin time prolongation was the only factor not previously included in other scores. Our multivarite analysis suggested that it was an independently associated factor to liver fibrosis. One limitation of this study is that the performance is only assessed in HBV-related patients. This is due to the high prevalence of HBV infection in Taiwan and thus a need to develop reliable fibrosis score for retrospective studies. Nevertheless, the four factors in the equation are not HBV-specific. Therefore, this score may be used to evaluate the fibrosis of other etiologies such as HCV infection and alcohol, once the corresponding validation is completed in these subjects.

The proposed HB-F score was constructed by multiple regression coefficients (Adjusted Beta). It is a challenge for both pathologists and fibrosis scores alike to discern the stage of early fibrosis (FS1 to FS3). This is also a limitation of HB-F. However, Figure 1A showed the distribution of HB-F score increased as the ISHAK-stage
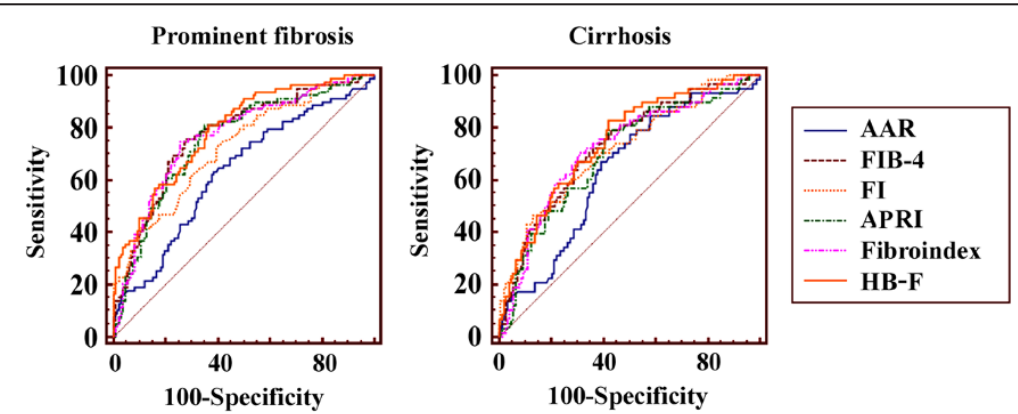

Figure 2 The ROC curves of HB-F score and 5 other methods (AAR, FIB-4, FI, APRI, and Fibroindex) for the diagnosis of prominent fibrosis (left) or cirrhosis (right) in the validation cohort. 

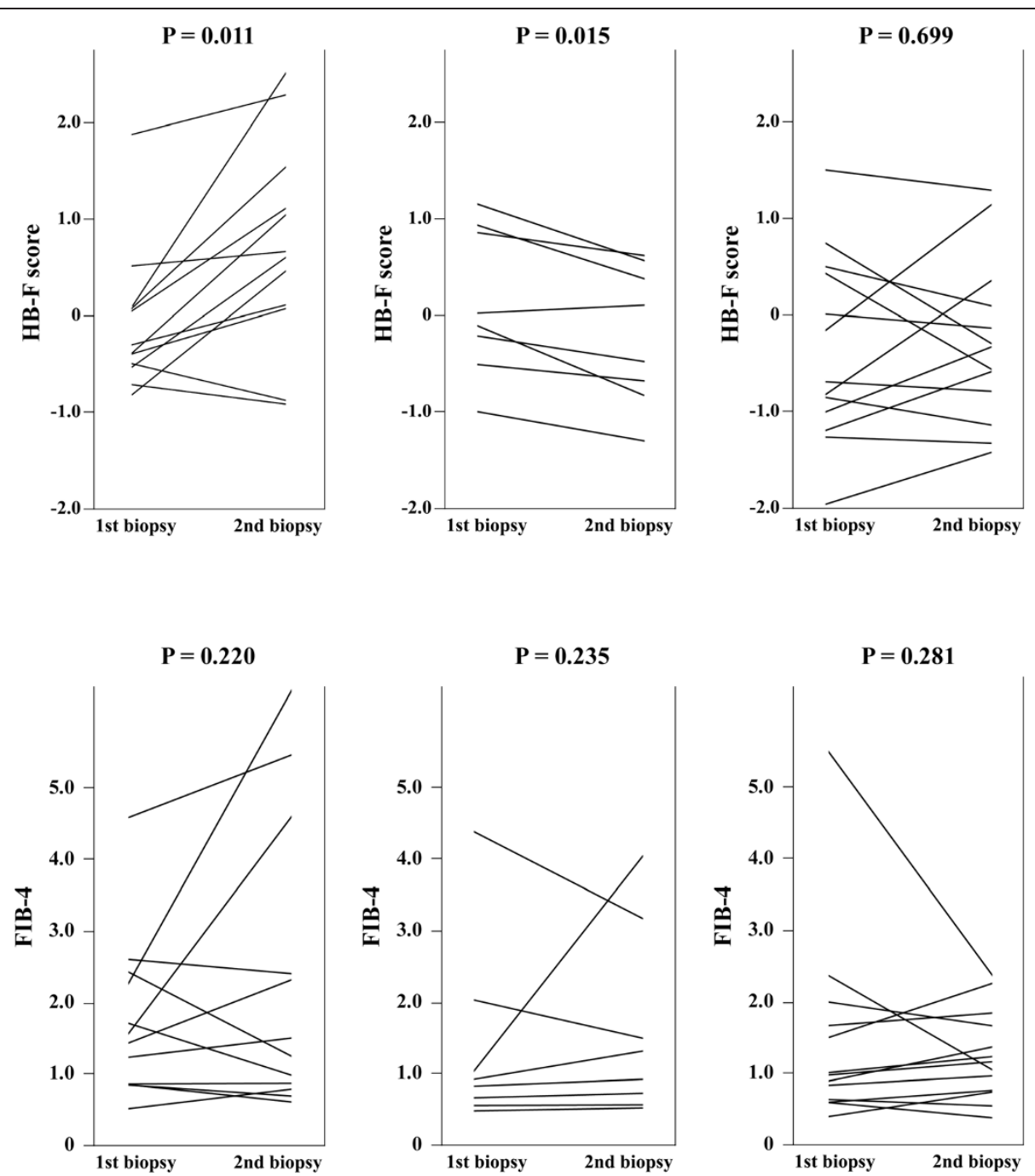

Figure 3 Comparison of HB-F and FIB-4 scores in determination of improvement or deterioration of liver fibrosis in paired biopsies. Left, The second biopsy showed an increase of ISHAK fibrosis level $(n=12)$. Middle, The second biopsy showed an decrease of ISHAK fibrosis level $(n=8)$. Right, The second biopsy showed the same ISHAK fibrosis level $(n=13)$.

ascended from FS1 to FS2. This observation suggested that HB-F might potentially be used for classifying the very earlier stage fibrosis. A larger number of patients in these two stages are needed for verification.

The HB-F score had superior performance than five other HCV-derived scores in identifying both the prominent fibrosis and cirrhosis patients, when assessed by AUCs. However, FIB-4, Fibroindex and APRI all showed very similar performance as in Figure 2 and Table 3, particularly FIB-4 seemed to be almost equally effective. However, when the scores were evaluated using paired liver biopsies, HB-F proved to be a better score for judgment of histology improvement or deterioration. Additionally, a similar performance between HB-F and other previous scores (FIB-4, FI, Fibroindex) suggested that we could use these scores interchangeably for estimation of fibrosis severity in retrospective studies where several clinical parameters were missing for one of these scores. In other words, our studies and the novel score provided flexibility for retrospective studies where fibrosis evaluation was often limited by data availability.

\section{Conclusions}

In conclusion, we have formulated a new fibrosis score, the HB-F, for chronic hepatitis B patients, by use of a linear combination of age, AST/ALT ratio, platelet count and prothrombin time prolongation values. Judging from the AUC comparison, HB-F score is better than other existing unpatented scores derived from hepatitis $C$ patients.

\section{Abbreviations}

AFP: Alpha-fetoprotein; ALT: Alanine transaminase; AST: Aspartate transaminase; AUC: Area under the curve; FI: Fibrosis index; GGT: Gamma glutamyl transpeptidase; HB-F: Hepatitis B-fibrosis; HBV: Hepatitis B virus;

HCV: Hepatitis C virus; ROC: Receiver operation characteristic. 


\section{Competing interests}

We declare that we have no competing interests.

\section{Authors contributions}

$\mathrm{CWH}$, designed and conducted the project, analyzed and interpreted the data, drafted the manuscript. $\mathrm{KHL}$, analyzed and interpreted the data, statistical analysis. SFH, handled tissue samples and interpreted the pathological data. KCT, handled blood samples and interpreted the serological data. CTY, medical oversight of the whole study implementation co-designed and supervised the conduct of the project, co-drafted and revised the manuscript. All authors read and approved the final manuscript.

\section{Author details}

'Liver Research Center, Chang Gung Memorial Hospital, 199, Tung Hwa North Road, Taipei, Taiwan. ${ }^{2}$ Department of Pathology, Chang-Gung Memorial Hospital, Taoyuan, Taiwan. ${ }^{3}$ Department of Medical Biotechnology and Laboratory Science, Research Center for Emerging Viral Infections, Chang Gung University, Taoyuan, Taiwan. ${ }^{4}$ Departments of Laboratory Medicine, Chang Gung Memorial Hospital, Taoyuan, Taiwan.

Received: 27 December 2012 Accepted: 23 May 2013

Published: 27 May 2013

\section{References}

1. Lavanchy D: Hepatitis B virus epidemiology, disease burden, treatment, and current and emerging prevention and control measures. J Viral Hepat 2004, 11:97-107.

2. Bataller R, Brenner DA: Liver fibrosis. J Clin Invest 2005, 41:1046-1055.

3. Ishak K, Baptista A, Bianchi L, et al: Histologic grading and staging of chronic hepatitis. J Hepatol 1995, 22:696-699.

4. Knodell RG, Ishak KG, Black WC, et al: Formulation and application of a numerical scoring system for assessing histological activity in asymptomatic chronic active hepatitis. Hepatology 1981, 1:431-435.

5. Bedossa P, Poynard T: An algorithm for the grading of activity in chronic hepatitis C. The METAVIR cooperative study group. Hepatology 1996 24:289-293.

6. Liaw YF, Sung JJ, Chow WC, et al: Lamivudine for patients with chronic hepatitis B and advanced liver disease. N Engl J Med 2004, 351:1521-1531.

7. Goodman ZD: Grading and staging systems for inflammation and fibrosis in chronic liver diseases. J Hepatol 2007, 47:598-607.

8. Ferrailoi G, Tinelli C, Malfitano A, et al: Performance of real-time strain elastography, transient elastography, and aspartate-to-platelet ratio index in the assessment of fibrosisi in chronic hepatitis C. Am J Roentgenol 2012, 199:19-25.

9. Kim SU, Lee $J H$, Kim Do Y, et al: Prediction of liver-related events using fibroscan in chronic hepatitis B patients showing advanced liver fibrosis. PLoS One 2012, 7:e36676.

10. Yoon KT, Lim SM, Park JY, et al: Liver stiffiness measurement using acoustic radiation force impulse (ARFI) elastography and effect of necroinflammation. Dig Dis Sci 2012, 57:1682-1691.

11. Cardoso AC, Carvalho-Filho RJ, Stern C, et al: Direct comparison of diagnostic performance of transient elastography in patients with chronic hepatitis B and chronic hepatitis C. Liver Int 2012, 32:612-621.

12. Williams $A L$, Hoofnagle $J H$ : Ratio of serum aspartate to alanine aminotransferase in chronic hepatitis: relationship to cirrhosis. Gastroenterology 1988, 95:734-739.

13. Wai CT, Greenson JK, Fontana RJ, et al: A simple noninvasive index can predict both significant fibrosis and cirrhosis in patients with chronic hepatitis C. Hepatology 2003, 38:518-526.

14. Ohta T, Sakaguchi K, Fujiwara A, et al: Simple surrogate index of the fibrosis stage in chronic hepatitis $C$ patients using platelet count and serum albumin level. Acta Med Okayama 2006, 60:77-84

15. Koda M, Matunaga $Y$, Kawakami M, et al: Fibrolndex, a practical index for predicting significant fibrosis in patients with chronic hepatitis $\mathrm{C}$. Hepatology 2007, 45:297-306

16. Sterling RK, Lissen E, Clumeck N, et al: Development of a simple noninvasive index to predict significant fibrosis in patients with HIV/HCV coinfection. Hepatology 2006, 43:1317-1325.

17. Vallet-Pichard A, Mallet V, Nalpas B, et al: FIB-4: an inexpensive and accurate marker of fibrosis in HCV infection. Comparison with liver biopsy and FibroTest. Hepatology 2007, 46:32-36.
18. Poynard T, Ngo Y, Munteanu M, et al: Biomarkers of liver injury for hepatitis clinical trial: a meta-analysis of longitudinal studies. Antivir The 2010, 15:617-631.

19. Stibbe KJ, Verveer C, Francke J, et al: Comparison of non-invasive assessment to diagnose liver fibrosis in chronic hepatitis B and $C$ patients. Scand J Gastroentero 2011, 46:962-972.

20. Poynard T, Ngo Y, Munteanu M, et al: Noninvasive markers of hepatitis fibrosis in chronic hepatitis B. Curr Hepat Rep 2011, 10:87-97.

21. Kim BK, Kim SU, Kim HS, et al: Prospective validation of FibroTest in comparison with liver stiffness for predicting liver fibrosis in Asian subjects with chronic hepatitis B. PLoS One 2012, 7:e35825.

\section{doi:10.1186/1756-0500-6-212}

Cite this article as: Hsu et al:: Development of a non-invasive fibrosis test for chronic hepatitis B patients and comparison with other unpatented scores. BMC Research Notes 2013 6:212.

\section{Submit your next manuscript to BioMed Central and take full advantage of:}

- Convenient online submission

- Thorough peer review

- No space constraints or color figure charges

- Immediate publication on acceptance

- Inclusion in PubMed, CAS, Scopus and Google Scholar

- Research which is freely available for redistribution 